\title{
SB365, Pulsatilla saponin D, suppresses the growth of gefitinib-resistant NSCLC cells with Met amplification
}

\author{
WON-JUN JANG $^{1 *}$, BYOUNGDUCK PARK $^{1 *}$, GIL-SAENG JEONG $^{1}$, SOON-SUN HONG ${ }^{2}$ and CHUL-HO JEONG ${ }^{1}$ \\ ${ }^{1}$ College of Pharmacy, Keimyung University, Daegu 704-701; ${ }^{2}$ Department of Biomedical Sciences, \\ College of Medicine, Inha University, Incheon, Republic of Korea
}

Received June 4, 2014; Accepted August 19, 2014

DOI: $10.3892 /$ or.2014.3528

\begin{abstract}
Clinical treatment using epidermal growth factor receptor-tyrosine kinase inhibitors (EGFR-TKIs) such as gefitinib or erlotinib has been applied in patients with non-small cell lung cancers (NSCLCs). Unfortunately, acquired drug resistance emerges in these patients due to the amplification of the Met proto-oncogene, which may be a compensatory mechanism of NSCLCs against EGFR inhibition. To overcome this resistance, identification of new small-molecule natural compounds is crucial for cancer therapeutics. In this regard, SB365, saponin D from the root of Pulsatilla koreana which has been used as a traditional medicine in Korea for several diseases, has attracted wide interest. In the present study, SB365 effectively suppressed the proliferation of gefitinibresistant HCC827GR NSCLC cells with Met amplification. Notably, our data revealed that SB365 inhibited the phosphorylation of Met and the downstream signaling pathway required for growth and survival in the Met-amplified HCC827GR cells. Moreover, SB365 suppressed the anchorage-independent growth, migration and invasion along with induction of apoptosis in the HCC827GR cells. Therefore, these results suggest that SB365 is good candidate as a natural product for use in the treatment of Met-amplified NSCLCs.
\end{abstract}

\section{Introduction}

Lung cancer is one of the most prevalent cancers and is the leading cause of death-related worldwide $(1,2)$. As non-small cell lung cancers (NSCLCs) account for almost $85 \%$ of all cases of human lung cancers, many therapeutic efforts have been made to treat NSCLCs $(3,4)$. During the last decade, receptor tyrosine kinases (RTKs) such as epidermal growth factor receptor (EGFR) have been regarded as appropriate targets for

Correspondence to: Professor Chul-Ho Jeong, College of Pharmacy, Keimyung University, Daegu 704-701, Republic of Korea

E-mail: chjeong75@kmu.ac.kr

${ }^{*}$ Contributed equally

Key words: SB365, saponin D, gefitinib, drug resistance, EGFR, Met
NSCLC treatment (5-7). Actually, aberrant overexpression of EGFR has been detected in $~ 50 \%$ of NSCLC patients and is known to be correlated with tumor growth and poor prognosis of various types of human cancers (8). Therefore, EGFRtyrosine kinase inhibitors (EGFR-TKIs) such as gefitinib or erlotinib have been developed for patients with NSCLCs or other human cancers $(9,10)$. However, despite the beneficial effect of these TKIs, EGFR-TKI therapies are now confronting the problem of acquired resistance (11). Recent studies have identified the two representative molecular mechanisms for this resistance: a secondary T790M mutation on exon 20 of the EGFR gene and amplification of the Met proto-oncogene or overexpression of hepatocyte growth factor (HGF) (12-16). Several EGFR-TKIs have been investigated to overcome this acquired resistance including irreversible EGFR-TKIs and TKIs selective for mutant EGFR (17). Moreover, combined treatment with EGFR-TKIs and Met-TKIs has been proposed to overcome resistance induced by c-Met amplification (18). Nonetheless, more effective and safer therapeutic agents for overcoming resistance to EGFR-TKIs are still needed.

Traditional natural compounds provide highly fertile ground for drug development, and a number of studies have proven that numerous purified natural products possess anticancer activities (19). Notably, Pulsatilla koreana is a traditional Korean herb which belongs to the family Ranunculaceae. Its roots have been widely used in traditional herbal medicine for the treatment of several diseases, in particular, malaria and amoebic dysentery in Korea (20). Notably, 17 types of saponins have been isolated from Pulsatilla koreana, among which, Pulsatilla saponin D (hereafter designated as SB365) has been reported to exert anticancer effects in several types of cancer cells (21-24). In particular, the present study demonstrated that SB365 inhibits c-Met phosphorylation by docking at its allosteric site, thereby suppressing the growth of gastric cancers (22). Based on this, it may be promising to assess the anticancer effects of SB365 in gefitinib-resistant Met-amplified NSCLCs.

In the present study, we assessed the anticancer efficacy of SB365 in Met-amplified HCC827GR cells. Here, we found that SB365 exerts its ability to inhibit anchorage-(in)dependent cell growth, migration and invasion by attenuating the Met-Akt signaling pathway in gefitinib-resistant HCC827GR cells. Moreover, our data also revealed that SB365 is sufficient for inducing apoptosis in gefitinib-resistant HCC827GR cells. 


\section{Materials and methods}

Materials. Antibodies for phospho-EGFR (Tyr1068), Met, phospho-Met (Tyr1234/1235), Akt, phospho-Akt (Ser473), Erk1/2, phospho-Erk1/2 (Thr202/Tyr204) and $\beta$-actin were purchased from Cell Signaling Technology (Beverly, MA, USA). Antibodies specific for EGFR and the secondary antibody were purchased from Santa Cruz Biotechnology (Santa Cruz, CA, USA). Gefitinib was purchased from LC Laboratories (Woburn, MA, USA). Halt ${ }^{\mathrm{TM}}$ Protease and phosphatase inhibitor cocktail (100X), EDTA (100X) and the BCA protein assay kit were purchased from Thermo Fisher Scientific (Rockford, IL, USA). 3-(4,5-Dimethylthiazol-2yl)2,5-diphenyltetrazolium bromide (MTT) was purchased from Sigma-Aldrich (St. Louis, MO, USA). PVDF membranes were purchased from Bio-Rad (Hercules, CA, USA). SuperSignal West Dura Extended Duration Substrate was purchased from Thermo Scientific (Waltham, MA, USA). A human phospho-RTK array kit was purchased from R\&D Systems (Minneapolis, MN, USA).

Cell culture. The human NSCLC cell line HCC827 was cultured in RPMI-1640 with L-glutamine supplemented with $10 \%$ fetal bovine serum (FBS) and penicillin/streptomycin. The gefitinib-resistant cell line HCC827GR was generated upon continuous exposure of parental HCC827 cells to gefitinib for over 6 months. In detail, starting with a gefitinib concentration of $10 \mathrm{nM}$, the exposure dose was doubled every 2 weeks until a final concentration of $10 \mu \mathrm{M}$ was achieved. HCC827GR cells were cultured in RPMI-1640 with L-glutamine supplemented with $10 \% \mathrm{FBS}$, penicillin/streptomycin and $1 \mu \mathrm{M}$ gefitinib. All cells were maintained at $37^{\circ} \mathrm{C}$ with $5 \% \mathrm{CO}_{2}$ in a humidifier incubator.

Preparation of SB365. SB365 was isolated from the roots of $P$. koreana, as previously described by Kim et al (25). Briefly, powered roots of $P$. koreana $(50 \mathrm{~g})$ were extracted 3 times with $50 \%$ aqueous ethanol $(500 \mathrm{ml})$, and the resulting extracts were combined and concentrated in vacuo to yield a light brown residue. The fraction was chromatographed using a Sephadex LH-20 column $(200 \mathrm{~g}, 60 \times 4 \mathrm{~cm})$ with an 80:20 mixture of methanol and $\mathrm{H}_{2} \mathrm{O}$, to give four fractions: SPX1 (139 mg, $24.8 \%)$, SPX2 (344 mg, 61.4\%), SPX3 (61 mg, 10.9\%) and SPX4 (15.7 mg, 2.8\%). After heating, the chromatogram was sprayed with $10 \% \mathrm{H}_{2} \mathrm{SO}_{4}$. The third fraction, which exhibited the most potent activity, was again chromatographed by solid phase HPLC [solid phase, RP-C18, 250 × $10 \mathrm{~mm}$; mobile phase, $\mathrm{MeOH}-\mathrm{H}_{2} \mathrm{O}$ (82:20); UV wavelength, $210 \mathrm{~nm}$; flow rate, $1 \mathrm{ml} / \mathrm{min}$ ] to yield 3 major fractions. Among them, the third fraction (SPX3), which exhibited the most potent activity, was purified by HPLC to yield saponin D, SB365. For more precise analysis of purified SB365, we also used mass spectrometry and NMR spectroscopy.

Cell proliferation assay. Cell proliferation was measured using MTT assay. HCC827 and HCC827GR cells were seeded at $2.5 \times 10^{3}$ cells/well in 96 -well plates. Following $24 \mathrm{~h}$ of incubation, the cells were treated with the indicated concentrations of the compounds, and subsequently incubated for 24, 48 and $72 \mathrm{~h}$ at $37^{\circ} \mathrm{C}$. After being incubated with the compounds, the medium was then replaced with medium containing MTT (0.5 mg/ml MTT, $100 \mu \mathrm{l} /$ well) and incubated for $4 \mathrm{~h}$. The medium was aspirated from the wells, and $100 \mu \mathrm{l}$ of dimethyl sulfoxide (DMSO) was added into each well and agitated for $180 \mathrm{sec}$. Absorbance at $565 \mathrm{~nm}$ was then read on a Tecan Infinite F200 Pro plate reader (Promega, Madison, WI, USA), and values are expressed as the percentage of absorbance from cells incubated in DMSO alone.

Western blot analysis. HCC827 and HCC827GR cells were seeded in a $100-\mathrm{mm}$ culture dish at $2 \times 10^{6}$ cells and incubated for $24 \mathrm{~h}$. The cells were treated with the indicated concentrations of the compounds or DMSO, and incubated for $24 \mathrm{~h}$. The cells were harvested in cold lysis buffer $(50 \mathrm{mM}$ Tris- $\mathrm{HCl} \mathrm{pH} 8.0$, $150 \mathrm{mM} \mathrm{NaCl}, 1 \%$ NP-40) with Halt ${ }^{\mathrm{TM}}$ Protease and phosphatase inhibitor cocktail and EDTA. The cells were lysed on ice for $30 \mathrm{~min}$ and centrifuged at $14,000 \mathrm{rpm}$ for $20 \mathrm{~min}$ at $4^{\circ} \mathrm{C}$. The proteins were quantified using the BCA method. Protein (20 $\mu \mathrm{l}$ ) was separated by SDS-PAGE and transferred to PVDF membranes. The membranes were blocked with $5 \%$ skim milk in $0.1 \% \mathrm{TBS}-\mathrm{T}$ at room temperature for $2 \mathrm{~h}$ with rocking and then incubated with the specific primary antibodies overnight at $4^{\circ} \mathrm{C}$. After washing the membrane with $0.1 \%$ TBS-T 3 times for $10 \mathrm{~min}$ each, the membranes were incubated with the HRP-conjugated secondary antibody at room temperature for $1 \mathrm{~h}$. The membranes were detected by SuperSignal West Dura Extended Duration Substrate. The membranes were imaged with LAS-3000 (Fuji, Japan) according to the manufacturer's instructions.

Phospho-RTK array analysis. Phospho-RTK array analysis was carried out using a human phospho-RTK array kit (R\&D Systems). Phospho-RTK array was performed according to the product manual. HCC827GR cells were seeded in a $100-\mathrm{mm}$ culture dish at $2 \times 10^{6}$ cells. The cells were treated with SB365 $(10 \mu \mathrm{M})$ and incubated for $24 \mathrm{~h}$. The cell lysates were prepared using NP40-lysis buffer containing the protease-phosphatase inhibitor cocktail and EDTA. After blocking for $1 \mathrm{~h}$ with Array Buffer 1, the arrays were incubated with $300 \mu \mathrm{g}$ of protein lysates overnight at $4^{\circ} \mathrm{C}$. The arrays were then washed and incubated with a HRP-conjugated phospho-tyrosine detection antibody. The arrays were detected by chemiluminescence and imaged using LAS-3000 according to the manufacturer's instructions. The intensity of the average signal of the pair of duplicated spots was calculated relative to the negative control spots.

Migration and invasion assay. Cell migration and invasion capacity in HCC827GR cells were assessed using BD cell-culture inserts and/or BD BioCoat Matrigel Invasion Chamber, respectively. The cells were suspended in culture media $\left(1 \times 10^{5}\right.$ cells $\left./ \mathrm{ml}\right)$ and then $0.5 \mathrm{ml}$ cell suspension $\left(5 \times 10^{4}\right.$ cells $\left./ \mathrm{ml}\right)$ was added to the upper chambers. After incubating for $24 \mathrm{~h}$ (for migration) or $48 \mathrm{~h}$ (for invasion), culture media in the inserts were carefully removed, and the membrane containing the cells on the lower surface of the inserts was fixed using methanol and stained with H\&E. The upper surface of the membrane was gently scrubbed with a cotton swab to remove the cells. The stained cells were quantified under a light microscope. 
Soft agar colony formation assay. HCC827GR cells $\left(8 \times 10^{3}\right.$ cells/well) were suspended in BME ( $1 \mathrm{ml}$ with $10 \% \mathrm{FBS}$, $0.33 \%$ agar) and plated over a layer of solidified bottom agar mixture (BME with 10\% FBS, 0.5\% agar) with the indicated concentrations of the drugs. The cultures were maintained at $37^{\circ} \mathrm{C}$ in a $5 \% \mathrm{CO}_{2}$ incubator for 6-7 days, and the number of colonies was counted under a light microscope.

Flow cytometry analysis. For assessing cell cycle distribution, the cells were detached and fixed overnight in ice-cold $70 \%$ ethanol. Fixed cells were washed with phosphate-buffered saline (PBS), and incubated with RNase A and propidium iodide (PI) at $37^{\circ} \mathrm{C}$ for $30 \mathrm{~min}$, and then cells were subjected to flow cytometric analysis to determine the percentage of cells at specific phases of the cell cycle. Apoptosis in HCC827GR cells was also measured using the Annexin V-FITC Apoptosis Detection Kit I (BD Biosciences Pharmingen) according to the product protocols. The cells were seeded in a $60-\mathrm{mm}$ culture dish at $5 \times 10^{5}$ cells. The cells were treated with various concentrations of the compounds and incubated for $24 \mathrm{~h}$. The cells were detached and then washed twice with cold PBS. The cells were suspended in $1 \mathrm{X}$ binding buffer $\left(5 \times 10^{5} \mathrm{cells} / \mathrm{ml}\right)$ and Annexin V-FITC and PI ( $5 \mu 1$ stock/100 $\mu$ l buffer) were added. After incubation at room temperature for $15 \mathrm{~min}$ in the dark, $400 \mu \mathrm{l}$ of $1 \mathrm{X}$ binding buffer was added to each tube. Flow cytometric analysis was performed using a BD FACSVerse flow cytometer and BD FACSuite software.

Statistical analysis. All quantitative data are presented as mean value \pm SD unless indicated otherwise. The statistical significance of compared measurements was measured using the Student's t-test, and $\mathrm{p}<0.05$ was considered to indicate a statistically significant result.

\section{Results}

Characterization of the gefitinib-resistant NSCLC cell line, HCC827GR. Gefitinib-resistant HCC827 cells (HCC827GR) were generated by continuously exposing the cells to increasing concentrations of gefitinib as reported $(14,26)$. The resultant HCC827GR cells were characterized by quantifying cell viability at different concentrations of gefitinib (Fig. 1A). In order to obtain this, the cells were incubated with the indicated concentration of gefitinib up to $72 \mathrm{~h}$, and cell proliferation was determined by MTT assay. As expected, our data showed that HCC827GR cells were resistant to gefitinib treatment when compared with the parental HCC827 cells (Fig. 1B). We further analyzed the protein expression and phosphorylation status of EGFR, Met and downstream signaling molecules in the HCC827 and HCC827GR cells. Our data clearly revealed that an abundant expression of Met protein was observed in the gefitinib-resistant HCC827GR cells, which was associated with emergence of acquired resistance (Fig. 1C). Unlike HCC827 cells, the phosphorylation status of EGFR, Met, Akt and Erk persisted at higher levels in the HCC827GR cells even following treatment with gefitinib (Fig. 1C). Therefore, our results indicate that gefitinib-resistant HCC827GR cells were successfully generated, and the bypass signaling mediated by Met led to persistent activation of downstream signaling in the HCC827GR cells in the presence of gefitinib.
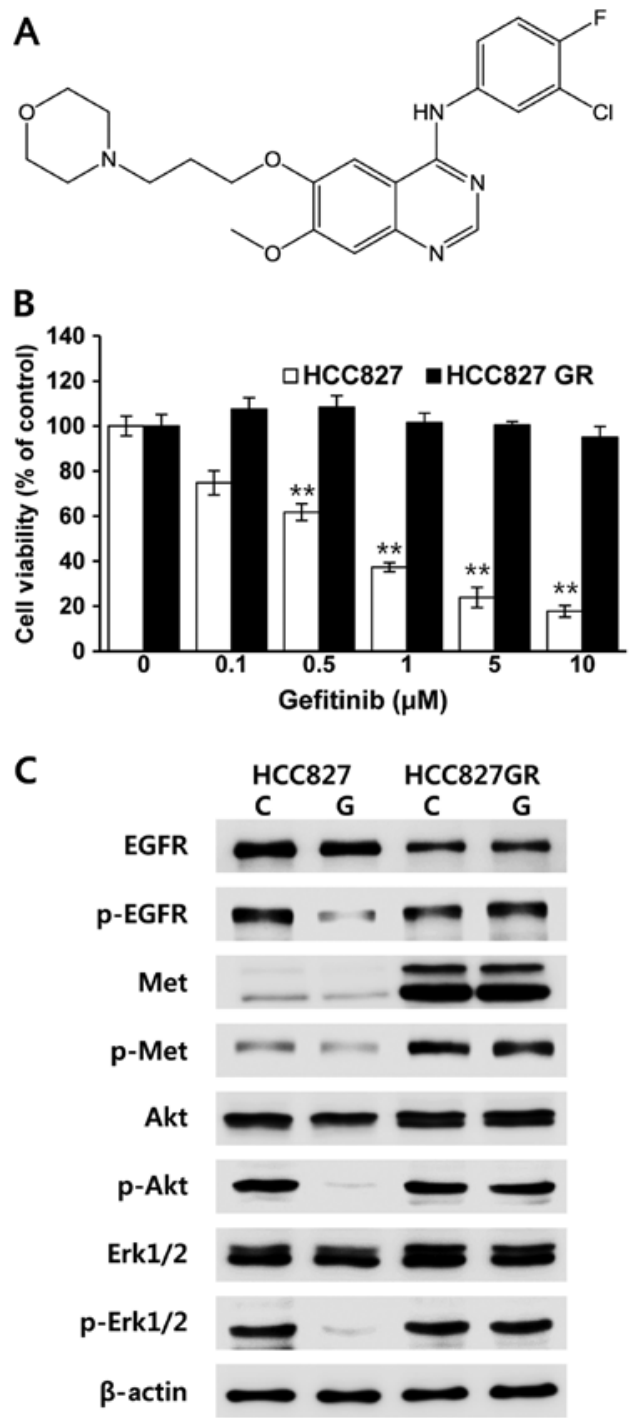

Figure 1. Characterization of the gefitinib-resistant HCC827GR cell line. (A) Chemical structure of gefitinib. (B) Gefitinib-sensitive (HCC827) and -resistant (HCC827GR) NSCLC cell lines were treated with various concentrations of gefitinib for 3 days, and cell viability was determined using the MTT assay. Data shown are representative of 3 independent experiments. Error bars represent the means \pm SD. Statistical significance was determined by the Student's t-test ( $\left.{ }^{* *} \mathrm{p}<0.01\right)$. (C) HCC827 and HCC827GR cell lines were treated with $1 \mu \mathrm{M}$ gefitinib for $24 \mathrm{~h}$. Whole cell lysates were assayed by western blot analysis using antibodies against total or the phosphorylated form of EGFR, Met and downstream proteins. $\beta$-actin was used as a loading control. C, control; G, gefitinib; EGFR, epidermal growth factor receptor.

SB365 inhibits anchorage-dependent and -independent growth of HCC $827 G R$ cells. To assess the inhibitory activity of SB365 (Fig. 2A) on the proliferation of gefitinib-resistant HCC 827GR cells, HCC827GR cells were treated with the indicated concentrations of SB365 up to $72 \mathrm{~h}$, and cell viability was determined by the MTT assay. Notably, treatment with SB365 decreased the cell viability of the HCC827GR cells in a dose-dependent manner (Fig. 2B), whereas co-treatment with gefitinib did not show any addictive or synergistic effects (data not shown). Next, we investigated the antitumor effect of SB365 in HCC $827 \mathrm{GR}$ cells by performing an anchorage-independent colony formation assay, which is an in vitro indicator and a key characteristic of the transformed cell phenotype (27). Our data revealed that treatment with SB365 resulted in fewer colonies 
A

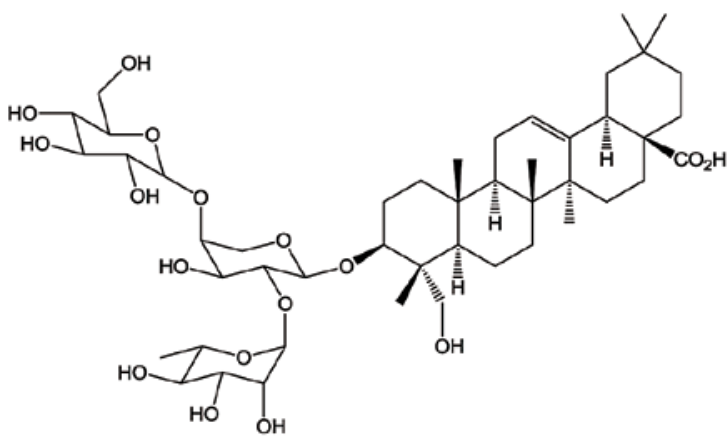

B

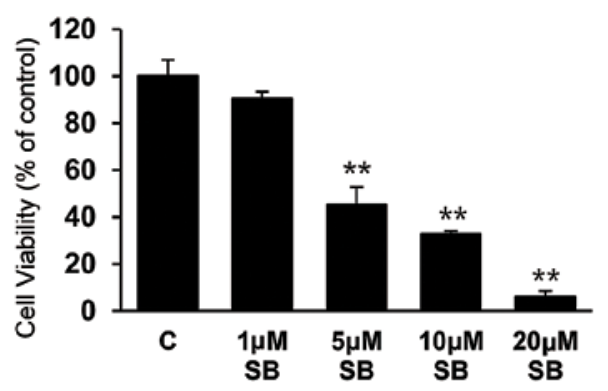

D

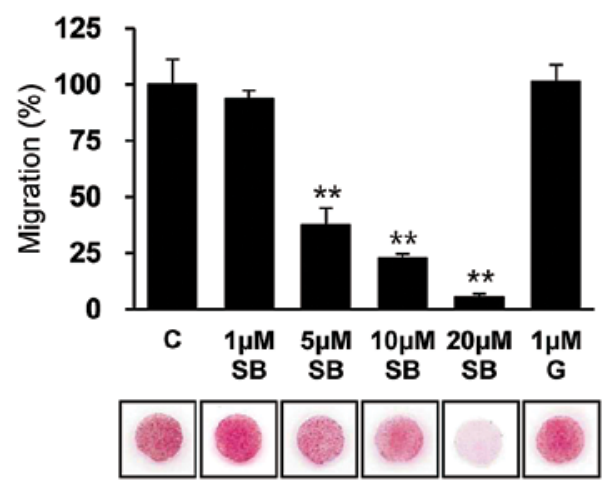

C

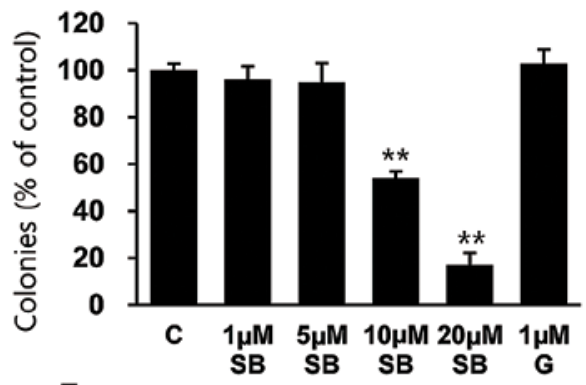

$\mathbf{E}$

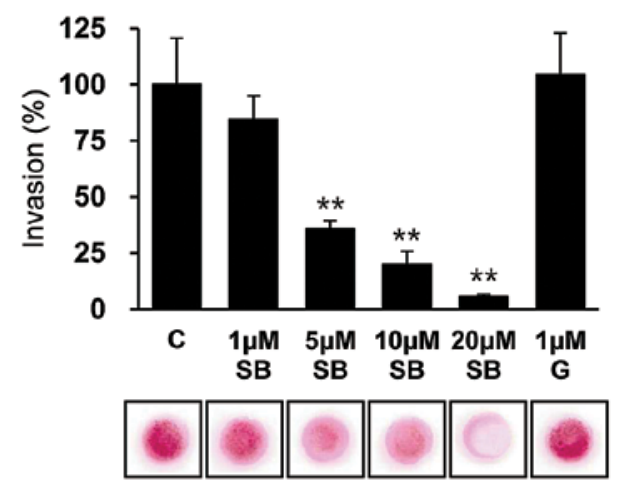

Figure 2. SB365 inhibits anchorage-independent cell growth, migration and invasion in gefitinib-resistant HCC827GR cells. (A) Chemical structure of SB365. (B) HCC 827GR cells were treated with various concentrations of SB365 for 3 days, and the cell viability was determined using the MTT assay. Data shown are representative of 3 independent experiments. Error bars represent the means \pm SD. (C) Colony formation ability of HCC827GR cells after exposure to SB365 for 7 days. Random areas were scanned (5 areas/well, 3-wells/set) in colonies grown in soft agar. Error bars represent the means \pm SD of 15 areas. (D) After incubation for $24 \mathrm{~h}$ (migration) or $48 \mathrm{~h}$ (invasion) with SB365, living cells that migrated or invaded to the lower surface were fixed, stained and counted using light microscopy. Random areas were scanned (5 areas/membrane of the well) in cells on the lower surface of the membrane in both the Boyden and Matrigel chamber. Error bars represent the means \pm SD of 5 areas. Statistical significance was determined by the Student's t-test ( $\left.{ }^{* *} \mathrm{p}<0.01\right)$. C, control; G, gefitinib; SB, SB365.

being formed in soft agar at concentrations of 10 and $20 \mu \mathrm{M}$ SB365 compared with the control cells or gefitinib-treated cells (Fig. 2C). These data indicate that treatment with SB365 inhibits both anchorage-dependent and -independent growth of HCC $827 \mathrm{GR}$ cells, suggesting that the malignant potential of HCC827GR cells is reduced by SB365 which may be a potential natural compound for overcoming acquired resistance to gefitinib.

Effects of SB365 on the migration and invasion of gefitinib-resistant HCC827GR cells. To investigate whether SB365 has antimetastatic activity in vitro, we performed migration and invasion assays in gefitinib-resistant HCC827GR cells. Our data showed that treatment with $1 \mu \mathrm{M}$ gefitinib did not affect the migratory and invasive capacities, whereas dose-dependent treatment with SB365 strongly inhibited the migratory and invasive capacities of the gefitinib-resistant HCC827GR cells up to $90 \%$ at a dose of $20 \mu \mathrm{M}$ (Fig. 2D and E). Therefore, these data revealed that SB365 has an antimetastatic effect on gefitinib-resistant HCC827GR cells.

SB365 inhibits the phosphorylation of Met and downstream signaling in HCC827GR cells. To explore the underlying mechanism by which SB365 inhibits anchorage-independent cell growth and in vitro metastatic capacities in gefitinib-resistant HCC827GR cells, we next performed a human phospho-RTK array to investigate the relative levels of phosphorylation of different RTKs. In agreement with a previous report (22), our data also revealed that SB365 may be a possible inhibitor of c-Met in NSCLCs. Notably, a decrease in phosphorylated-Met 

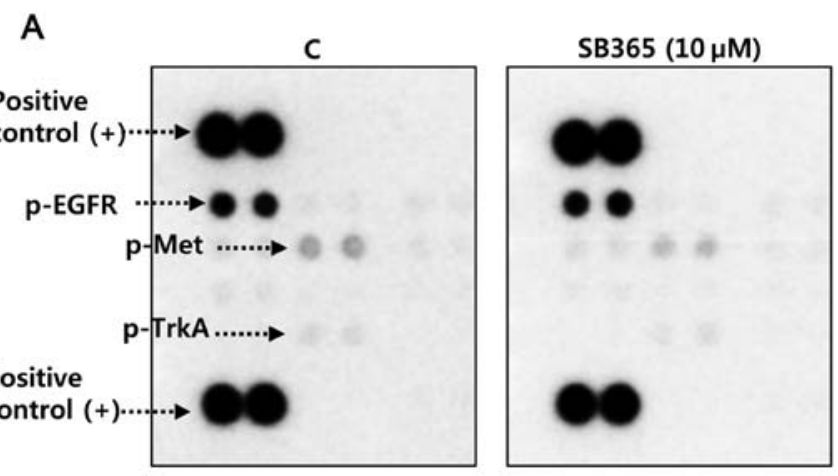

B

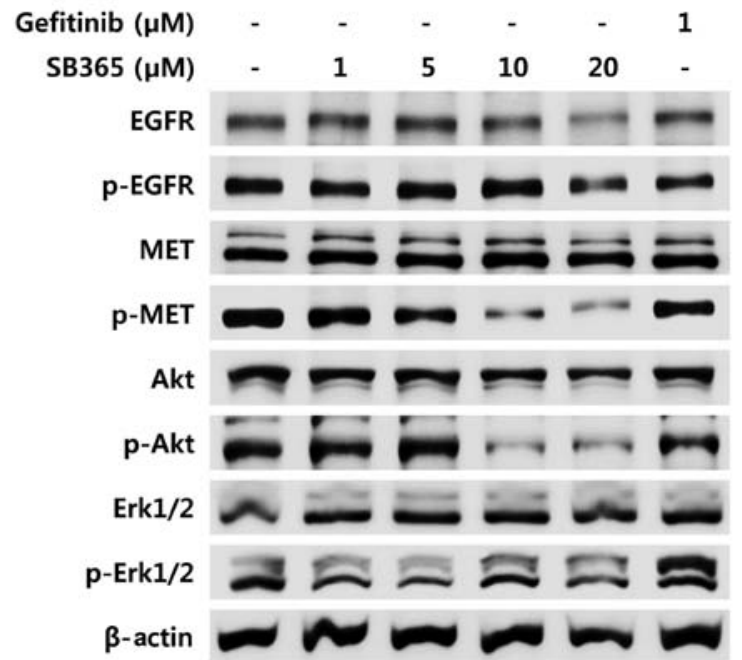

Figure 3. SB365 inhibits the phosphorylation of Met and downstream signaling in gefitinib-resistant HCC827GR cells. (A) Phosphorylation status of several RTKs by SB365. Phospho-RTK array analysis was carried out using a human phospho-RTK array kit. (B) HCC827GR cells were treated with the indicated concentration of drugs for $24 \mathrm{~h}$. Whole cell lysates were assayed by western blot analysis using antibodies against total or the phosphorylated form of EGFR, Met and downstream proteins. $\beta$-actin was used as a loading control. RTKs, receptor tyrosine kinases; EGFR, epidermal growth factor receptor.

was observed in the HCC827GR cells after SB365 treatment, whereas the phosphorylation level of EGFR was not changed in the $10 \mu \mathrm{M}$ SB365-treated HCC827GR cells (Fig. 3A). To better understand the mechanism responsible for c-Met inhibition by SB365, we examined the protein expression and phosphorylation status of RTK proteins including EGFR, Met and downstream signaling molecules in the HCC827GR cells. In order to obtain this, cells were treated with $1 \mu \mathrm{M}$ of gefitinib or various concentrations of SB365 at 1, 5, 10 and $20 \mu \mathrm{M}$ for $24 \mathrm{~h}$. As expected, treatment with $1 \mu \mathrm{M}$ gefitinib did not affect the phosphorylation status of EGFR, Met, Akt and Erk1/2 in the gefitinib-resistant HCC827GR cells (Fig. 3B). When HCC827GR cells were treated with various concentrations of SB365, the phosphorylation levels of Met and downstream signaling molecule Akt but not Erk1/2 were effectively suppressed at doses of 10 or $20 \mu \mathrm{M}$ SB365 (Fig. 3B). Therefore, these findings indicate that SB365 suppressed cancer progression through the Met-Akt signaling pathway in Met-amplified NSCLC HCC827GR cells.
SB365 induces apoptosis in the HCC827GR cells. To determine the pro-apoptotic effect of SB365 on gefitinib-resistant HCC827GR cells, flow cytometric analysis was performed. An increased proportion of apoptotic cells in the sub-G1 phase was strongly detected following dose-dependent treatment with SB365 (4.9-13.5\%) compared with the control $(2.5 \%)$, indicating a pro-apoptotic effect of SB365 in the HCC827GR cells (Fig. 4A). Moreover, flow cytometric analysis with Annexin V showed that treatment with SB365 induced early apoptosis (Annexin $\mathrm{V}^{+} / \mathrm{PI}^{-}$) in the HCC827GR cells, yet not with gefitinib (Fig. 4B). A few late apoptotic cells (Annexin $\mathrm{V}^{+} /$ $\mathrm{PI}^{+}$) were also observed in the cells treated with $20 \mu \mathrm{M}$ SB365 (Fig. 4B). More importantly, its apoptotic effect was accompanied by increased levels of cleaved caspase- 3 and poly(ADP ribose) polymerase (Fig. 4C). Moreover, treatment with SB365 increased the protein expression levels of cleaved caspase-3 and PARP and decreased Bcl-2 expression (Fig. 4C). These findings clearly suggest that treatment with SB365 is sufficient for inducing death signaling even in gefitinib-resistant NSCLC HCC827GR cells with Met amplification.

\section{Discussion}

To date, reversible small-molecule EGFR-TKIs, including gefitinib (Iressa ${ }^{\circledR}$ ) and erlotinib (Tarceva ${ }^{\circledR}$ ), have been recommended as molecular-targeted anticancer drugs for patients with NSCLC with EGFR-activating mutations $(9,10)$. However, despite excellent initial responses to EGFR-TKIs in these NSCLC patients, nearly all patients eventually develop drug resistance after a median period of $\sim 10$ months through two genetically conferred mechanisms; a secondary T790M mutation in EGFR and c-Met gene amplification (12-16). For this reason, a strategy using irreversible EGFR-TKIs or the combined use of EGFR- and Met-TKIs has been proposed to overcome resistance to EGFR-TKIs. Considering this, it is crucial to establish an alternative treatment strategy overcoming resistance to EGFR-TKIs to improve the survival of patients with NSCLC.

A recent trend in anticancer therapy has been centered on the search for anticancer drugs which are safer and highly patient acceptable. Consistently, the use of medicinal compounds from herbal/natural sources is becoming increasingly popular over synthetic drugs as an alternative treatment choice for cancer patients (28). Based on this notion, we isolated SB365, a saponin D from Pulsatilla koreana and explored its anticancer effects on gefitinib-resistant NSCLC cells. Previous results suggest that saponins have been known to possess a wide range of anticancer properties such as chemopreventive or chemotherapeutic effects on various types of cancer cells $(29,30)$. We also found that SB365, a saponin D, exerted remarkable anticancer properties in several types of cancer cells including hepatocellular carcinoma, gastric, colon and pancreatic cancers (21-24). In the present study, we showed that SB365 has an inhibitory effect on the anchorage-independent proliferation, migration and invasion of HCC827GR NSCLC cells resistant to gefitinib. Moreover, SB365 was sufficient for inducing apoptosis in gefitinib-resistant HCC827GR cells. These effects of SB365 against gefitinib resistance may be attributed to its ability to inhibit both Met phosphorylation and subsequent Akt activation in Met-amplified HCC827GR cells. 
A
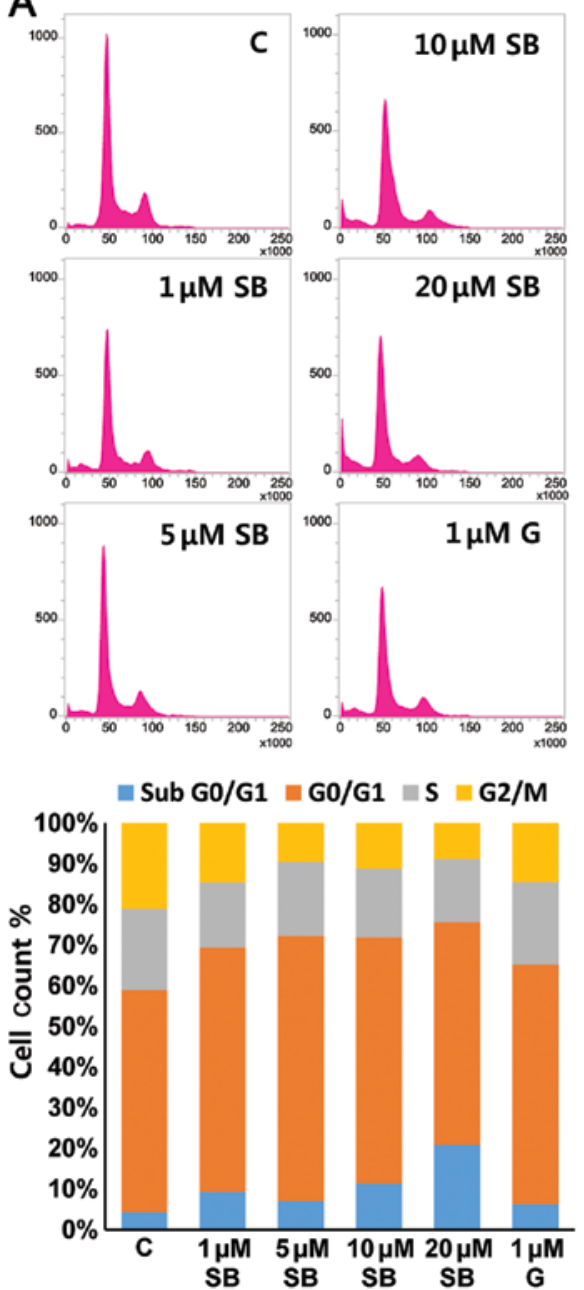

B
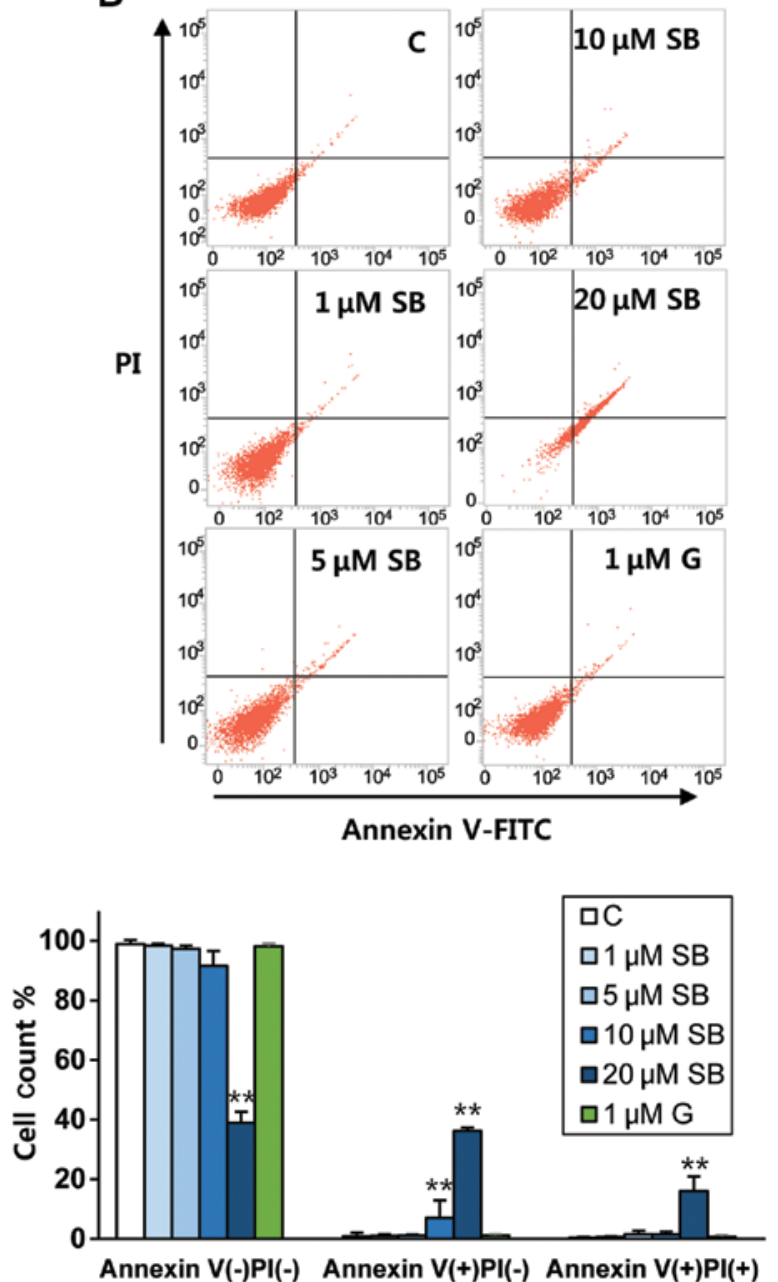

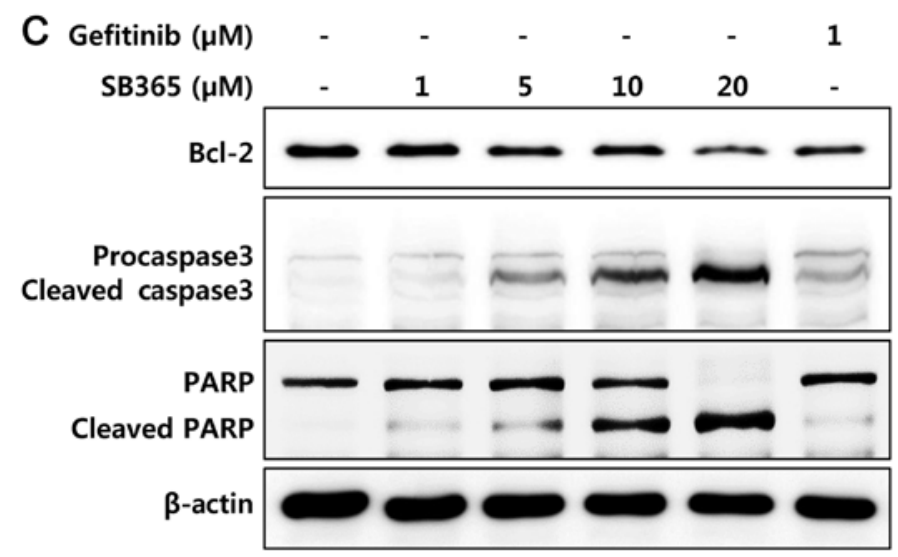

Figure 4. SB365 induces apoptosis in gefitinib-resistant HCC827GR cells. (A) HCC827GR cells were treated with SB365, and cellular DNA was stained with propidium iodide (PI) and flow cytometric analysis was performed to analyze the cell cycle distribution. The percentage of the cell population in each phase is indicated as a graph. (B) Flow cytometric analysis with Annexin V staining was performed in SB365-treated HCC827GR cells. Flow cytometric analysis was performed using a BD FACSVerse flow cytometer and BD FACSuite software. Data shown are representative of 3 independent experiments. Error bars represent the means $\pm \mathrm{SD}$. Statistical significance was determined by the Student's t-test ( $\left.{ }^{* *} \mathrm{p}<0.01\right)$. C, control; G, gefitinib; SB, SB365. (C) Whole cell lysates were assayed by western blot analysis after exposure to the indicated concentrations of SB365 in HCC827GR cells.

Accumulated evidence suggests that identification of natural compounds with high anticancer efficacy and defined molecular targets could be a prerequisite for successful drug discovery. For example, various phytochemicals, including [6]-gingerol (31), resveratrol (32) and myricetin (33), have been reported to directly interact with their targets in cells, thereby inhibiting cancer cell proliferation. Consistent with this, we previously reported that SB365 effectively inhibited the phosphorylation of Met by binding to an allosteric site of Met in MKN-45 gastric cancers, indicating that SB365 may be a potential natural inhibitor of Met (22). Met is a well-established mediator of carcinogenesis and induces an invasive 
program consisting of cell proliferation, migration, invasion and survival (34). In addition, Met activation prevents cancer cell apoptosis through the PI3K-Akt signaling pathway (35). Based on the notion that EGFR-TKI resistance can be accompanied by Met amplification, SB365 may be effectively used for the treatment of Met-amplified NSCLCs.

In the present study, we generated gefitinib-resistant HCC827GR cells with Met amplification and then evaluated the anticancer activity of SB365. The present data demonstrated that SB365 inhibited anchorage-independent proliferation, migration, and invasion of HCC827GR cells and induced cancer cell apoptosis by a caspase-dependent manner. Moreover, our phospho-array data revealed that the phosphorylation of Met, not EGFR, was robustly decreased by treatment with SB365 in HCC827GR cells. Collectively, our findings suggest that SB365 is a new therapeutic candidate to overcome resistance to EGFR-TKIs in NSCLCs harboring Met amplification.

\section{Acknowledgements}

This study was conducted by the Settlement Research Grant of Keimyung University in 2011 (to C-H.J.).

\section{References}

1. Jung KW, Park S, Kong HJ, et al: Cancer statistics in Korea: incidence, mortality and survival in 2006-2007. J Korean Med Sci 25: 1113-1121, 2010.

2. Siegel R, Naishadham D and Jemal A: Cancer statistics, 2013. CA Cancer J Clin 63: 11-30, 2013.

3. Owonikoko TK, Ragin CC, Belani CP, et al: Lung cancer in elderly patients: an analysis of the surveillance, epidemiology, and end results database. J Clin Oncol 25: 5570-5577, 2007.

4. Sher T, Dy GK and Adjei AA: Small cell lung cancer. Mayo Clin Proc 83: 355-367, 2008

5. Fukuoka M, Yano S, Giaccone G, et al: Multi-institutional randomized phase II trial of gefitinib for previously treated patients with advanced non-small-cell lung cancer (The IDEAL 1 Trial) [corrected]. J Clin Oncol 21: 2237-2246, 2003.

6. Hotta K, Matsuo K, Ueoka H, Kiura K, Tabata M and Tanimoto M: Addition of platinum compounds to a new agent in patients with advanced non-small-cell lung cancer: a literature based metaanalysis of randomised trials. Ann Oncol 15: 1782-1789, 2004.

7. Shepherd FA, Rodrigues Pereira J, Ciuleanu T, et al: Erlotinib in previously treated non-small-cell lung cancer. N Engl J Med 353: 123-132, 2005.

8. Laskin JJ and Sandler AB: Epidermal growth factor receptor: a promising target in solid tumours. Cancer Treat Rev 30: 1-17, 2004.

9. Maemondo M, Inoue A, Kobayashi K, et al: Gefitinib or chemotherapy for non-small-cell lung cancer with mutated EGFR. N Engl J Med 362: 2380-2388, 2010.

10. Mitsudomi T, Morita S, Yatabe Y, et al: Gefitinib versus cisplatin plus docetaxel in patients with non-small-cell lung cancer harbouring mutations of the epidermal growth factor receptor (WJTOG3405): an open label, randomised phase 3 trial. Lancet Oncol 11: 121-128, 2010.

11. Rosell R, Moran T, Queralt C, et al: Screening for epidermal growth factor receptor mutations in lung cancer. N Engl J Med 361: 958-967, 2009.

12. Pao W, Miller VA, Politi KA, et al: Acquired resistance of lung adenocarcinomas to gefitinib or erlotinib is associated with a second mutation in the EGFR kinase domain. PLoS Med 2: e73, 2005.

13. Bean J, Brennan C, Shih JY, et al: MET amplification occurs with or without $T 790 M$ mutations in EGFR mutant lung tumors with acquired resistance to gefitinib or erlotinib. Proc Natl Acad Sci USA 104: 20932-20937, 2007.
14. Engelman JA, Zejnullahu K, Mitsudomi T, et al: MET amplification leads to gefitinib resistance in lung cancer by activating ERBB3 signaling. Science 316: 1039-1043, 2007.

15. Yano S, Wang W, Li Q, et al: Hepatocyte growth factor induces gefitinib resistance of lung adenocarcinoma with epidermal growth factor receptor-activating mutations. Cancer Res 68: 9479-9487, 2008.

16. Sequist LV, Waltman BA, Dias-Santagata D, et al: Genotypic and histological evolution of lung cancers acquiring resistance to EGFR inhibitors. Sci Transl Med 3: 75ra26, 2011.

17. Engelman JA, Zejnullahu K, Gale CM, et al: PF00299804, an irreversible pan-ERBB inhibitor, is effective in lung cancer models with $E G F R$ and $E R B B 2$ mutations that are resistant to gefitinib. Cancer Res 67: 11924-11932, 2007.

18. Tang Z, Du R, Jiang S, et al: Dual MET-EGFR combinatorial inhibition against T790M-EGFR-mediated erlotinib-resistant lung cancer. Br J Cancer 99: 911-922, 2008.

19. Saha SK and Khuda-Bukhsh AR: Molecular approaches towards development of purified natural products and their structurally known derivatives as efficient anti-cancer drugs: current trends. Eur J Pharmacol 714: 239-248, 2013.

20. Youn HJ, Lakritz J, Kim DY, Rottinghaus GE and Marsh AE: Anti-protozoal efficacy of medicinal herb extracts against Toxoplasma gondii and Neospora caninum. Vet Parasitol 116: $7-14,2003$.

21. Hong SW, Jung KH, Lee HS, et al: SB365 inhibits angiogenesis and induces apoptosis of hepatocellular carcinoma through modulation of PI3K/Akt/mTOR signaling pathway. Cancer Sci 103: 1929-1937, 2012.

22. Hong SW, Jung KH, Lee HS, et al: SB365, Pulsatilla saponin D, targets c-Met and exerts antiangiogenic and antitumor activities. Carcinogenesis 34: 2156-2169, 2013.

23. Son MK, Jung KH, Hong SW, et al: SB365, Pulsatilla saponin D suppresses the proliferation of human colon cancer cells and induces apoptosis by modulating the $\mathrm{AKT} / \mathrm{mTOR}$ signalling pathway. Food Chem 136: 26-33, 2013.

24. Son MK, Jung KH, Lee HS, et al: SB365, Pulsatilla saponin D suppresses proliferation and induces apoptosis of pancreatic cancer cells. Oncol Rep 30: 801-808, 2013.

25. Kim Y, Bang SC, Lee JH and Ahn BZ: Pulsatilla saponin D: the antitumor principle from Pulsatilla koreana. Arch Pharm Res 27: 915-918, 2004.

26. Ghosh G, Yan X, Lee AG, Kron SJ and Palecek SP: Quantifying the sensitivities of EGF receptor (EGFR) tyrosine kinase inhibitors in drug resistant non-small cell lung cancer (NSCLC) cells using hydrogel-based peptide array. Biosens Bioelectron 26: 424-431, 2010.

27. Freedman VH and Shin SI: Cellular tumorigenicity in nude mice: correlation with cell growth in semi-solid medium. Cell 3: 355-359, 1974.

28. Zhao J: Nutraceuticals, nutritional therapy, phytonutrients, and phytotherapy for improvement of human health: a perspective on plant biotechnology application. Recent Pat Biotechnol 1: 75-97, 2007.

29. Taylor WG, Elder JL, Chang PR and Richards KW: Microdetermination of diosgenin from fenugreek (Trigonella foenum-graecum) seeds. J Agric Food Chem 48: 5206-5210, 2000.

30. Price KR, Johnson IT and Fenwick GR: The chemistry and biological significance of saponins in foods and feedingstuffs. Crit Rev Food Sci Nutr 26: 27-135, 1987.

31. Jeong CH, Bode AM, Pugliese A, et al: [6]-Gingerol suppresses colon cancer growth by targeting leukotriene $\mathrm{A}_{4}$ hydrolase. Cancer Res 69: 5584-5591, 2009.

32. Oi N, Jeong $\mathrm{CH}, \mathrm{Nadas} \mathrm{J}$, et al: Resveratrol, a red wine polyphenol, suppresses pancreatic cancer by inhibiting leukotriene $\mathrm{A}_{4}$ hydrolase. Cancer Res 70: 9755-9764, 2010.

33. Jung SK, Lee KW, Byun S, et al: Myricetin inhibits UVB-induced angiogenesis by regulating PI-3 kinase in vivo. Carcinogenesis 31 : 911-917, 2010.

34. Birchmeier C, Birchmeier W, Gherardi E and Vande Woude GF: Met, metastasis, motility and more. Nat Rev Mol Cell Biol 4: 915-925, 2003.

35. Xiao GH, Jeffers M, Bellacosa A, Mitsuuchi Y, Vande Woude GF and Testa JR: Anti-apoptotic signaling by hepatocyte growth factor/Met via the phosphatidylinositol 3-kinase/Akt and mitogen-activated protein kinase pathways. Proc Natl Acad Sci USA 98: 247-252, 2001. 